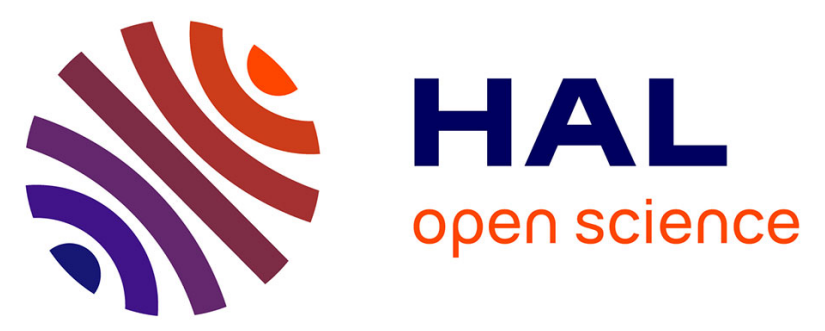

\title{
Classification structurale des divers composes fluores du fer et de cations monovalents. Application a leurs proprietes magnetiques
}

\author{
Josik Portier, Alain Tressaud, Robert de Pape, Paul Hagenmuller
}

\section{To cite this version:}

Josik Portier, Alain Tressaud, Robert de Pape, Paul Hagenmuller. Classification structurale des divers composes fluores du fer et de cations monovalents. Application a leurs proprietes magnetiques. Materials Research Bulletin, 1968, 3 (5), pp.433-436. hal-01944483

\section{HAL Id: hal-01944483 \\ https://hal.science/hal-01944483}

Submitted on 4 Dec 2018

HAL is a multi-disciplinary open access archive for the deposit and dissemination of scientific research documents, whether they are published or not. The documents may come from teaching and research institutions in France or abroad, or from public or private research centers.
L'archive ouverte pluridisciplinaire HAL, est destinée au dépôt et à la diffusion de documents scientifiques de niveau recherche, publiés ou non, émanant des établissements d'enseignement et de recherche français ou étrangers, des laboratoires publics ou privés. 


\section{CLASSIFICATION STRUCTURALE DES DIVERS COMPOSES FLUORES DU FER ET DE CATIONS MONOVALENTS. APPLICATION A LEURS PROPRIETES MAGNETIQUES.}

par MM. J. PORTIER, A. TRESSAUD, R. de PAPE et P. HAGENMULLER (Service de chimie minérale structurale de la Faculté des Sciences de BORDEAUX associé au C. N.R.S., 351, cours de la Libération, TALENCE, Gironde, FRANCE

et Laboratoire de chimie minérale du C.S.U. du MANS, route de Laval, LE MANS, Sarthe, France).

\section{ABSTRACT}

The structures of the phases occuring in the $\mathrm{FeF}_{3}-\mathrm{FeF}_{2}-\mathrm{MF}$ $(M=L i, N a, K, R b, T l)$ systems differ in the manner in which the $\mathrm{FeF}_{6}$ octahedra are connected. These structures may assume the form of : a)tridimensional $\left(\mathrm{FeF}_{3}\right)_{\mathrm{n}}$ networks, b) double layers of $\left.\left(\mathrm{Fe}_{2} \mathrm{~F}_{7}\right)_{n}, \mathrm{c}\right)$ single layers of $\left.\left(\mathrm{FeF}_{4}\right)_{n}, \mathrm{~d}\right)$ bridged chains of $\left(\mathrm{Fe}_{3} \mathrm{~F}_{14}\right)_{n}$, e) isolated chains of $\left(\mathrm{FeF}_{5}\right)_{n}$ or f) isolated $\mathrm{FeF}_{6}$ octahedra. This classification, which can be extended to other compounds of transition elements, aluminium and gallium, accounts for the magnetic properties of the iron fluorocompounds.

Nous avons décrit dans des mémoires antérieurs les systèmes $\mathrm{FeF}_{3}-\mathrm{FeF}_{2}-\mathrm{MF}(\mathrm{M}=\mathrm{Li}, \mathrm{Na}, \mathrm{K}, \mathrm{Rb}, \mathrm{Tl})(1,2,3)$. La détermination des réseaux cristallins des diverses phases observées permet de dégager une classification structurale qui rappelle celle des borates ou des silicates. Cette systématique s'appuie sur trois règles essentielles :

- le fer, qu'il soit au degré d'oxydation deux ou trois, possède toujours la coordinence 6 ,

- les octaèdres $\left(\mathrm{FeF}_{6}\right)$ sont liés par leurs sommets; ils n'ont ni arêtes ni faces communes,

- tous les atomes de fluor participent à la formation de ces 
octà̀dres.

Les phases de formulation $\mathrm{M}_{\mathrm{x}} \mathrm{FeF}_{3}(0<\mathrm{x}<1)$ sont caractérisées par un réseau tridimensionnel dans lequel tous les octaèdres ( $\mathrm{FeF}_{6}$ ) ont leurs sommets communs. Ce sera le cas du fluorure $\mathrm{FeF}_{3}$ luimême (4), des perovskites $\mathrm{KFeF}_{3}(5), \mathrm{RbFeF}_{3}$ (6), $\mathrm{TlFeF}_{3}$ (3), de $\mathrm{NaFeF}_{3}$ is otype de $\mathrm{GdFeO}_{3}$ (7), ainsi que des bronzes fluorés $\mathrm{M}_{\mathrm{x}} \mathrm{FeF}_{3}(\mathrm{M}=\mathrm{Na}, \mathrm{K}, \mathrm{Rb}, \mathrm{Tl})(1,3)$, dont les structures sont analogues à celles des bronzes oxygénés de tungstène $\mathrm{M}_{x} \mathrm{WO}_{3}$.

$\mathrm{K}_{3} \mathrm{Fe}_{2} \mathrm{~F}_{7}$, seul composé connu de ce type, est constitué de doubles couches d'octaèdres de composition $\left(\mathrm{Fe}_{2} \mathrm{~F}_{7}\right)_{\mathrm{n}}(2)$. Chaque octaèdre est lié à ses voisins par cinq de ses sommets.

$\mathrm{K}_{2} \mathrm{FeF}_{4}, \mathrm{KFeF}_{4}$, les variétés basse température $\alpha$ et haute température $\beta$ de $\mathrm{RbFeF}_{4}, \mathrm{Rb}_{2} \mathrm{FeF}_{4}$ sont caractérisés par des couches simples $\left(\mathrm{FeF}_{4}\right)_{\mathrm{n}}$ d'octaèdres séparés par des couches d'ions alcalins. Les octaèdres sont donc liés par quatre de leurs sommets.

$$
\mathrm{Na}_{5} \mathrm{Fe}_{3} \mathrm{~F}_{14}(8) \text {, isotype de la chiolite, comporte des couches }
$$

$\left(\mathrm{Fe}_{3} \mathrm{~F}_{14}\right)_{\mathrm{n}}$ formées de files pontées d'octaèdres. Un tiers des octaèdres est lié à ses voisins par quatre sommets communs, deux tiers le sont par deux seulement.

$\mathrm{Rb}_{2} \mathrm{FeF}_{5}$ est sans doute isotype de $\mathrm{Tl}_{2} \mathrm{AlF}_{5}$. Il serait alors constitué de chaînes d'octaèdres $\left(\mathrm{FeF}_{5}\right)_{\mathrm{n}}$ mettant deux sommets en commun.

$$
\mathrm{Na}_{3} \mathrm{FeF}_{6} \text { isotype de la cryolithe d'une part, } \mathrm{K}_{3} \mathrm{FeF}_{6}, \mathrm{Rb}_{3} \mathrm{FeF}_{6}
$$

(9) et $\mathrm{Tl}_{3} \mathrm{FeF}_{6}$ isotypes de $\left(\mathrm{NH}_{4}\right)_{3} \mathrm{AlF}_{6}$ d'autre part sont caractérisés par des octaèdres $\mathrm{FeF}_{6}$ isolés.

La Fig. 1 schématise les divers types structuraux observés.

L'expérience montre que cette classification peut être étendue aux

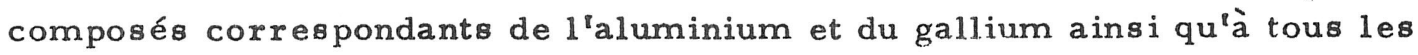
éléments de transition du titane au zinc. Signalons cependant que $\mathrm{Na}_{2} \mathrm{CuF}_{4}$, cont la structure a été déterminée par D, Babel, fait exception (11). En effet dans ce composé chaque octaèdre est lié par deux de ses arêtes aux octaèdres voisins.

Elle rend compte également des propriétés magnétiques des di- 


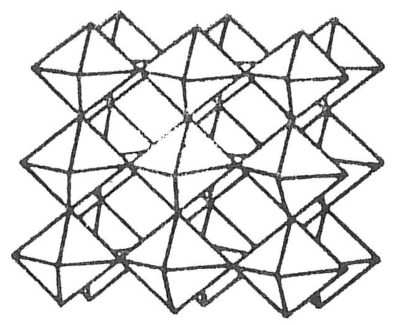

$\left(\mathrm{FeF}_{3}\right)$

$$
\left[F \bullet F_{3}, M F_{e} F_{3}, M_{x} F \bullet F_{3}\right]
$$
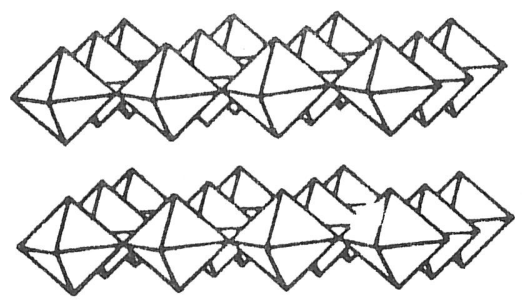

$\left(\mathrm{FeF}_{4}\right)$

$\left[\mathrm{MFeF}_{4}, \mathrm{M}_{2} \mathrm{~F} \times \mathrm{F}_{4}\right]$

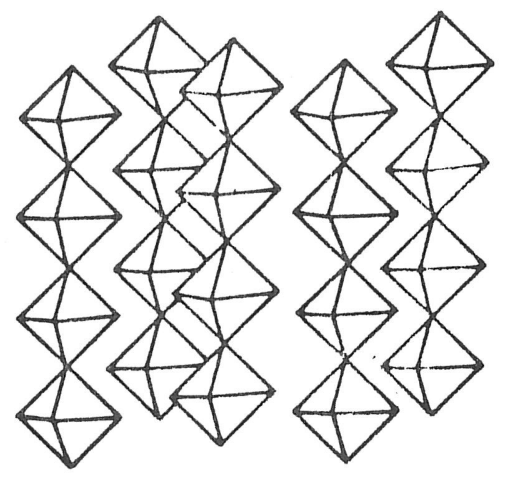

$\left(\mathrm{FeF}_{5}\right)$

$\left[\mathrm{M}_{2} \mathrm{Fe} \mathrm{F}_{5}\right]$
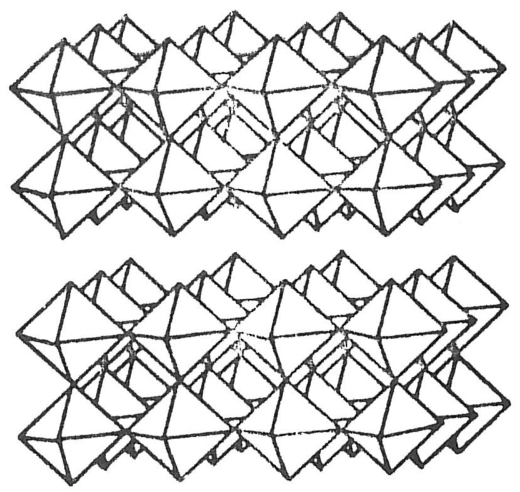

$\left(\mathrm{Fe}_{2} \mathrm{~F}_{7}\right)$

$\left[\mathrm{K}_{3} \mathrm{Fe}_{2} \mathrm{~F}_{7}\right]$

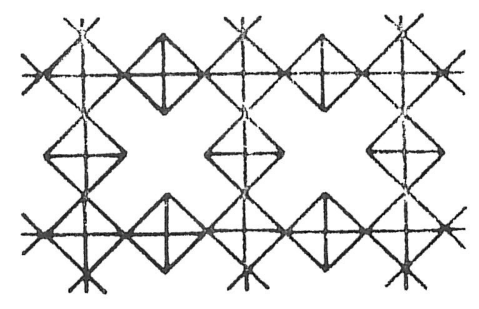

$$
\begin{gathered}
\left(\mathrm{Fe}_{3} \mathrm{~F}_{14}\right) \\
{\left[\mathrm{No}_{5} \mathrm{Fe}_{3} \mathrm{~F}_{14}\right]}
\end{gathered}
$$

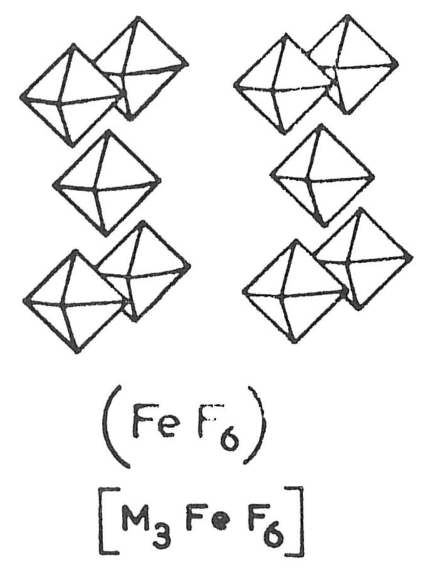

FIG. 1

Représentation idéalisée des diverses structures des composés fluorés du fer et des cations monovalents. 
vers composés du fer étudiés. On peut prévoir en effet, pour les phases dans lesquelles le rapport $\frac{F}{F e}$ est faible, des couplages magnétiques importants en raison de la proximité des atomes de fer. $\mathrm{KFeF}_{3}(10)$ et $\mathrm{TlFeF}_{3}$ sont effectivement antiferromagnétiques avec des points de Néel situés respectivement à $120^{\circ} \mathrm{K}$ et $100^{\circ} \mathrm{K}$. La Fig. 1 montre que seul $\mathrm{Na}_{5} \mathrm{Fe}_{3} \mathrm{~F}_{14}$ possède deux types de sites cristallographiques différents; $1^{\prime} u n$ des sous-réseaux contient deux fois plus d'atomes de fer que l'autre; on peut donc prévoir des propriétés ferrimagnétiques. Knox a effectivement montré que $\mathrm{Na}_{5} \mathrm{Fe}_{3} \mathrm{~F}_{14}$ est ferrimagnétique à basse température avec un point de Curie à $80^{\circ} \mathrm{K}(8)$. On peut s'attendre enfin à ce que les couplages magnétiques soient faibles pour un rapport $\frac{F}{F e}$ élevé, tout particulièrement lor sque les octaèdres sont isoles. $\mathrm{Na}_{3} \mathrm{FeF}_{6}$ et $\mathrm{Tl}_{3} \mathrm{FeF}_{6}$ ne sont effectivement que paramagnétiques. Ils suivent une loi de Curie-Weiss avec des températures de Curie respectivement de $-12^{\circ} \mathrm{K}$ et $-6^{\circ} \mathrm{K}(12)$.

\section{Références}

1.R. de Pape, C.R.Acad.Sc., 260, 4527, 1965.

2. R. de Pape, Bull. Soc. Chim.Fr, 12, $3489,1965$.

3. A. Tressaud, Thèse de $3^{\circ}$ Cycle, Bordeaux 1967.

4. F. Ebert, Z, anorg, allg, Chem., 196, 395, 1931.

5. K. Knox, Acta Cryst., 14, 583, 1961.

6. M. Kestigian, F.D. Leipziger, W. J. Croft, R. Guidoboni, Inorg. Chem, $\underline{5}, 1462,1966$.

7. D. J. Machin, R.S. Nyholm, J.Chem.Soc., $1798,1954$.

8. K. Knox, S. Geller, Phys.Rev., 110, $771,1958$.

9. H. Bode, E. Voss, Z. anorg.allg. Chem., 290, 1, 1957.

10. D. J. Machin, R. Martin, R. S. Nyholm, J. Chem. Soc., $1490,1963$.

11. D. Babel, Z. anorg.allg. Chem, 336, 200, 1965.

12. Les mesures magnétiques ont été effectuées au laboratoire d'Electrostatique et de Physique du Métal de Grenoble, dans le service de M. le Professeur Pauthenet. 